\title{
Chitosan and Textile Industry
}

\author{
Mehrnaz Moattari ${ }^{1}$, Farahnaz Moattari², Homa Mohseni Kouchesfehani ${ }^{1 *}$, Gholamreza Kaka ${ }^{3 *}$, Seyed Homayoon \\ Sadraie $^{3}$ and Majid Naghdi ${ }^{4}$
}

${ }^{1}$ Department of Animal Biology, Faculty of Biological Science, Kharazmi University, Tehran, Iran

${ }^{2}$ Faculty of Agriculture and Natural Resources, Persian Gulf University, Bushehr, Iran.

${ }^{3}$ Neuroscience Research Center, Baqiyatallah University of Medical Sciences, Tehran, Iran

${ }^{4}$ Fasa University of Medical Science, Fasa, Fars, Iran

*Corresponding author: Homa Mohseni Kouchesfehani, Department of Animal Biology, Faculty of Biological Science, Kharazmi University, P0Box: 15719-14911, Tehran, Iran

Gholamreza Kaka, Neuroscience Research Center, Baqiyatallah University of Medical Sciences, Aghdasie, Artesh Boulevard, Artesh Square, P0Box: 19568-37173,Tehran, Iran

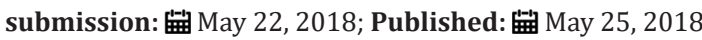

\begin{abstract}
Based on the origin, textiles are categorized into natural and synthetic materials. Chitosan is the deacetylated form of chitin. Chitosan is the second most abundant polysaccharide in nature after cellulose, and has been extensively used in agriculture, cosmetics, food and nutrition, environmental protection, and material sciences. This biomaterial is a promising topic of research for future studies concerning biomedical textiles. Chitosan has been studied for a number of useful properties such as biocompatibility, biodegradability, anti-inflammatory, wound healing, antitumor effects and antibacterial properties in most available biomaterials have been tested in different approaches by different groups. Here, the purpose is to mention the application of chitosan, a biopolymer, and chitosan derivatives in medical textiles and purposes.
\end{abstract}

Keywords: Chitosan; Medicine; Industry; Textile

\section{Introduction}

Undoubtedly, fibres play very important functional roles in human being lives so that it is difficult today to define a field where a fibre doesn't involve. In fact, fibres cover a wide range including living muscles, the bandages, automobile tyres, river guards, etc. [1]. Notably, in the field of biomedical sciences researches involving the developments in the usage of textile fibres is concentrated [2]. Nowadays, there has been increased attention towards environmentally friendly, biocompatible and biodegradable polymers, as most of the widespread synthetic polymers lack these possessions. Hence, the benefits of chitosan as a textile fibre were appreciated [3]. For instance, it is unaffected by thermal disintegration and can act as a suitable drug delivery carrier. It also exhibits the permeability and selectivity required for medical purposes. It can provide better control of transport and inherent blood compatibility [4].

\section{Discussion}

\section{Chitin}

Chitin, a natural biopolymer, is the second most abundant polysaccharide in nature after cellulose. Chitin is embedded in a protein matrix of a crustacean shell or a squid pen. It is institute the shells of crustaceans such as crabs, shrimps and lobster, in the exoskeleton of insects and molluscs and in the cell walls of certain fungi. For the first time, Braconnot in 1811 dicovered and isolated chitin and named it fungine [5]. Later, Odier [6] found chitin in insects and called it as chitin [6]. Nowadays, chitin is commonly derived from crab and shrimp shells which are discarded as massive wastes by the marine industry. The crustacean exoskeleton waste comprises $30-50 \%$ calcium carbonate, $30-40 \%$ protein and $20-30 \%$ chitin on a dry mass foundation [7].

\section{Production of chitosan}

Chitosan is a cationic polysaccharide prepared from alkaline $\mathrm{N}$-deacetylation of chitin. Therefore, chitosan is the group name dedicated to the polymers that are derived from deacetylation of chitin. The only difference between chitosan and chitin is the degree of deacetylation [3]. For the first time, Rouget in 1859 discovered chitosan by reaction of chitin with sodium hydroxide solution that leads to hydrolyze the $\mathrm{N}$-acetyl linkages or deacetylation of chitin [8]. Later, Rigby [9] achieved two patents, one for chitosan making from chitin and other for production of films and fibres from chitosan. Chitosan is a cationic polysaccharide prepared from alkaline N-deacetylation of chitin. Chitosan has been extensively used in agriculture, cosmetics, food and nutrition and environmental protection and has anti-infection, anti- 
bacterial, antitumor, antiulcer, immunostimulatory, antibacterial, haemostatic, non-toxicity and adsorption [10] and wound-healing properties. Definitely, life sciences and technologies is the territory where chitosan and chitosan derivatives have much attention as a biomedical material, due to its prominent biological functions. Indeed, recent analyses evidently showed that the interest in chitosan in the life sciences and technology lead to enhance and to receive funding by research agencies worldwide. Here, the authors offer an overview of the state of the art of a wide range of chitosan and materials derived from them which can be utilized in various fields of life sciences and technologies. Chitosan has a spongy property which can be used in localized drug delivery as a cell scaffold or carrier to control the release of growth factors to the site of injury. It has also another roles such as DNA condensation, food microbiology, and crop protection [11].

\section{Application of chitosan in textiles}

Fibres have extensive applications in wearable and decorative textiles. In the past decades, chitosan has been appraised for various textile usages. Use of chitosan in textile industries consists of fabrication of manufactured fibres [12], textile (cotton, silk, wool, polypropylene) dyeing [13], finishing including silk dyeing [5,14] and wool dyeing [15]. [282- 283], polypropylene dyeing [16], durable press finishing [17], wool finishing [18]. [296-304], antimicrobial finishing [19], antistatic finishing [20], de-odourising finishing [21], sizing [22], textile printing [23], textile ink jet printing [24] and medical textiles.

\section{Chitosan fibres as medical textile}

Chitosan is achieved approval as a textile fibre which can be definitely used for medical purposes, despite the problems faced in its process-ability and inferior mechanical properties. It is an exclusive biomaterial with positive distinctive properties, favored for medical usages. In fact, chitosan has intra-molecular hydrogen bonding formed between the hydroxyl and amino groups. So, it is easy to handle because of its thermal resistance and is used in medicine. Chitosan fibres can be used effectively for absorbable sutures and wound dressings. It is reported that using chitosan as a wound dressings enhance effectively the rate of healing by $75 \%$. Since chitosan has permeability and selectivity properties, it can be used effectively in artificial kidney systems and artificial skin as a membrane. N-Carboxyalkyl (aryl) chitosan, N-Carboxyacyl chitosan, o-Carboxyalkyl chitosan, Sugar derivatives and Metal ion chelates are some examples of chitosan derivatives with potential applications in medicine. For example, $\mathrm{N}$-acylchitosan can be used in textiles membrane and medical aids [25]. These unique textile membranes requisite to be establish for improvement of transportation, ease of formability and blood compatibility [4].

\section{Conclusion}

Application of chitosan in textile industry and especially medical membranes and aids is gaining more popularity nowadays and coming decades due to its eco-friendly properties.

\section{Acknowledgement}

We thank Prof. Hedayat Sahraei, respected head of Neuroscience Research Center, Baqiyatallah University of Medical Sciences, Tehran, Iran, because of his kind considerations.

\section{References}

1. Kumar PS, Suganya S (2017) Introduction to sustainable fibres and textiles. Sustainable Fibres and Textiles, Elsevier, pp. 1-18.

2. Kumar B, Mahaboobi S, Satyam S (2017) Chitosan in medicine-A mini review. J Mol Pharm Org Process Res 5(134): 2.

3. Moattari M (2018) Chitosan-film associated with mesenchymal stem cells enhanced regeneration of peripheral nerves: A rat sciatic nerve model. J Chemical Neuroanat 88: 46-54.

4. Pavinatto A (2017) Chemically modified chitosan derivatives. Frontiers in Biomaterials: Chitosan Based Materials and its Applications 3: 107132 .

5. Wu Y (2003) The effect of chitosan and its derivatives on the dyeability of silk. The Hong Kong Polytechnic University, China.

6. Odier A (1823) Memory on the branchiobdelle, new kind of annelids of the family Hirudinées.

7. Mathew S (2017) Aquaceuticals for developing high value noble foods and dietary supplements. Indian Journal of Agricultural Biochemistry 30(1): 1-9.

8. Raymond L, Morin FG, Marchessault RH (1993) Degree of deacetylation of chitosan using conductometric titration and solid-state NMR. Carbohydrate Research 246(1): 331-336.

9. Rigby GW (1936) Substantially undegraded deacetylated chitin and process for producing the same. Google Patents.

10. Xie W, Xu P, Liu Q (2001) Antioxidant activity of water-soluble chitosan derivatives. Bioorganic \& Medicinal Chemistry Letters 11(13): 16991701.

11. Sarmento B (2011) Chitosan and chitosan derivatives for biological applications: chemistry and functionalization. International Journal of Carbohydrate Chemistry.

12. Kumar MR (1999) Chitin and chitosan fibres: a review. Bulletin of Materials Science 22(5): 905.

13. Metha R, Combs R (1991) An improved process for NEP coverage in dyeing cotton. Am Dyest Rep 80(9): 74-79.

14. Hu Z, Chan W, Szeto Y (2006) Dyeing silk with chitosan nanoparticles. J Asia Text Apparel.

15. Yen MS (2001) Application of chitosan/nonionic surfactant mixture in reactive dyes for dyeing wool fabrics. Journal of Applied Polymer Science 80(14): 2859-2864.

16. Rochery M, Lam T, Crighton J (1997) FTIR\&ATR analyses on a polypropylene (pp) surface after plasma treatment in the study of chitosan surface grafting to improve PP dyeing behaviour. Macromolecular Symposia 119(1): 277-282.

17. Bhattacharyya N (1993) Novel formaldehyde scavengers in resin finish of cotton fabrics. American Dyestuff Report 83(3): 96.

18. Rybicki E (2000) Application of natural biopolymers in shrink-proofing of wool. Fibres and Textiles in Eastern Europe 8: 62-65.

19. Lim SH (2003) Synthesis of a fiber-reactive chitosan derivative and its application to cotton fabric as an antimicrobial finish and a dyeingimproving agent. 
20. Lim SH, Hudson SM (2003) Review of chitosan and its derivatives as antimicrobial agents and their uses as textile chemicals. Journal of Macromolecular Science, Part C: Polymer Reviews 43(2): 223-269.

21. Bandyopadhyay B (1999) Development of eco-friendly preservative for textile wet processing. BTRA SCAN 30(4): 1-7.

22. Hasebe Y, Kuwahara K, Tokunaga S (2001) Applied technology chitosan hybrid deodorant agent for finishing textiles-A new polymeric deodorizer exhibited effective deodorant performance against human malodors. AATCC Review-American Association of Textile Chemists and Colorists 1(11): 23-27.
23. Abou-Okeil A, Hakeim O (2005) Effect of metal ion binding of chitosan on the printability of pretreated wool fabric. Coloration Technology 121(1): 41-44.

24. Yuen C (2008) Digital ink-jet printing of cotton fabric with chitosan. Textile Asia.

25. Muzzarelli RA (1983) Chitin and its derivatives: new trends of applied research. Carbohydrate Polymers 3(1): 53-75.

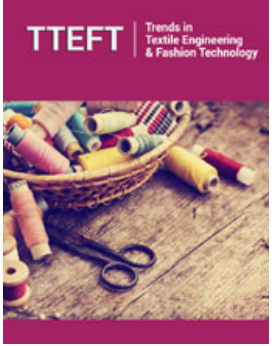

Trends in Textile Engineering \& Fashion Technology

\section{Benefits of Publishing with us}

- High-level peer review and editorial services

- Freely accessible online immediately upon publication

- Authors retain the copyright to their work

- Licensing it under a Creative Commons license

- Visibility through different online platforms 